\title{
Performance Analysis of FrFT based Adaptive Filters with LMS Algorithm
}

\author{
K.Yashas Kumar \\ M.Tech Student \\ Department of Electronics and \\ Communications, \\ Aditya Institute of Technology \\ and Management, \\ Tekkali, Srikakulam, AP, India
}

\author{
P.V.Muralidhar \\ Associate professer, \\ Department of Electronics and \\ Communications, \\ Aditys Institute of Technology \\ and Management, \\ Tekkali, Srikakulam,AP, India
}

\author{
D.V.L.N.Sastry \\ Assistant Professer, \\ Department of Electronics and \\ Instrumentation, \\ Aditya Institute of Technology \\ and Management, \\ Tekkali,Srikakulam, AP, India
}

\begin{abstract}
Fractional Fourier Transform function $(\mathrm{FrFT})$ is a generalization of Fourier transforms (FT). Finite Impulse Response (FIR) filters are implemented based on Fractional Fourier transform domains, then modified filters characteristics some what tunable when compare with existing FT based FIR filters. So, in our proposals implementation is made on the FrFT FIR filters for different windows like Rectangle, Bartlett, Hamming, Hanning, and Kaiser, based on the adaptive algorithms , and the performance of the proposed filters is made by SNR values of the different obtain filters for given noisy sinusoidal inputs.
\end{abstract}

\section{Keywords}

Adaptive Filters, FrFT, LMS Algorithm, SNR

\section{INTRODUCTION}

Digital filter plays an important role in digital signal processing applications. Digital filters are widely used in digital signal processing applications, such as digital signal filtering, noise filtering, signal frequency analysis, speech and audio compression, biomedical signal processing and image enhancement etc.[1]. Traditionally, most digital filter applications have been limited to audio and high-end image processing. With advances in process technologies and digital signal processing methodologies, digital filters are now costeffective in the IF range and in almost all video markets[2].Finite impulse response (FIR) digital filter impulse response is finite, so it can be used for Fast Fourier Transform (FFT) algorithm to achieve the filtered signal, which can greatly improve the efficiency of operation. In addition, FIR digital filter can be designed a linear phase digital filter which is convenient for image processing and data transmission applications[3]

FIR Filter banks are used to perform short-term spectram Analysis in a variety of speech processing systems[4]. Many Window functions are widely used in digital signal processing for various applications in signal analysis and estimation, digital filter design and speech processing [5]. Embedded devices. Heart rate frequency is very important health status information. The frequency measurement is used in many medical or sport applications like stress tests or life treating situation prediction.[6]. The accurate extraction of the AA signal from the ECG of AF is of great interest for subsequent analysis, since it has been documented to provide significant information on the properties of $\mathrm{AF}$ episodes[7]. reduction represents another important objective of ECG signal processing; in fact, the waveforms of interest are sometimes so heavily masked by noise that their presence can only be revealed once appropriate signal rocessing has first been applied.[8] In the last two decades, spectral analysis of the residual ECG signal (rECG, i.e. an ECG signal in which ventricular components were canceled through beat averaging techniques) has been employed to characterize atrial activities[9].VF is raditionally described as a system of many chaotic in the myocardium wandering, electrical wavelets, ever changing in direction and number In contrast, recent findings indicate that stable organized centres of rapid activity, called "mother rotors"[10].

\section{ADAPTIVE FILTERS \& LMS ALGORITHM}

Adaptive filters provide performance excellence due to their inherent pole-zero structure as compared with adaptive finite impulse response (FIR) filters that have an all-zero form ,in active noise control Application[1].RLS Filters[2].Adaptive Filters are highly stable and effectively attenuate and often cancel destitutions[3].An Adaptive filters are successfully used in bio-medical processing systems like Denoising of ECG Waveforms[4] Adaptive filters play an important role in modern Digital signal processing products in area such as telephone echo cancellations, noise cancellation, equalization of communications channels, biomedical signal enhancement, active noise control, and adaptive control systems [5]

\section{FRACTIONAL FOURIER TRANSFORM}

Fractional Fourier Transform widely used in quantum mechanics and quantum optics [16].Fractional Fourier Analysis can obtain the mixed time and frequency components of signals[17].it finds various applications like pattern recognisition with some spatial distortion, Image representation, compression and noise removal in signal processing [18]-[20].FrFT used for Interpretation of sinusoidal signals and design of Digital FIR Filters[21]-[22].

The continuous -time Fractional Fourier Transform of a signal $\omega(t)$ is defined through an interval [3]

$$
\omega_{\alpha}(u)=\int_{-\infty}^{\infty} \omega(t) K_{\alpha}(t, u) d t---(1)
$$

Where the transform kernel $K_{\alpha}(t, u)$ of the FRFT is Given by 


$$
\begin{array}{r}
K_{\alpha}(t, u)=\sqrt{\frac{1-j \cot (\alpha)}{2 \pi}} \exp \left[i\left(\frac{t^{2}+u^{2}}{2}\right) \cot (\alpha)\right. \\
-\operatorname{iutcosec}(\alpha)] \text { if } \alpha \text { is multiple of } \pi \\
=\partial(t-u) \text { if } \alpha \text { is multiple of } 2 \pi
\end{array}
$$

$=\partial(t+u)$ if $\alpha+\pi$ is a mulpiple of $2 \pi---(2)$

Where $\alpha$ indicates rotation of angle of the Transformed signal for FrFT.

\section{DESIGN OF FRFT BASED ADAPTIVE FILTER}

In our illustrative numerical example, the adaptive filter is set to be a 100-tap FrFT based FIR filter to simplify numerical algebra. The filter adjustable coefficient wn is adjusted based on the LMS algorithm.

$$
w_{n+1}=w_{n}+m * e(n) x(n)---(3)
$$

where wn is the coefficient used currently, while $w_{n+1}$ is the coefficient obtained from the LMS algorithm and will be used for the next coming input sample. The value of $m$ controls the speed of the coefficient change, $e(n)$ is an error value updated each time and $x(n)$ is noised signal coefficient. The output equations of LMS algorithm leads to

$$
\begin{aligned}
& F(n)=G(n) * x(n)---(4) \\
& e(n)=d(n)-y(n)---(5) \\
& G_{n+1}=G+m * e(n) x(n)--(6)
\end{aligned}
$$

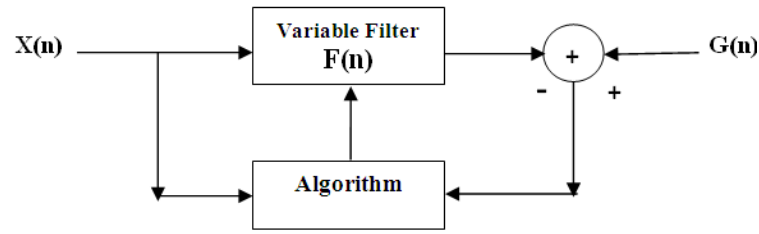

\subsection{Steps to Design Adaptive Filter}

1. The Low pass filter removes the corrupting low frequency noises in signal. The order of the filter is the order of the filter is 64 .

\section{Steps to low pass filter}

The desired transfer function of filter is

$$
h_{d}(n)=\frac{\sin _{c} n}{\pi n}
$$

By multiplying the desired transfer function with windows designed by FrFT, we can get transfer function of FIR band reject filter i.e

$$
h(n)=h_{d}(n) * w(n)
$$

where $w(n)$ is represents Transfer function of following windows

\footnotetext{
i Hamming window

ii Hanning window

iii Kaiser window
}

2. Now $h(n)$ is compared with $x(n)$ which produces $e(n)$.

3. The error coefficients are fed back to LMS algorithm to update the coefficients of FrFt based LPF.

4. Steps 2 and 3 repeated up to error becomes negligible.

5. The updated coefficients of LMS Algorithm is the is the Response of desired Filter

\section{RESULTS AND IMPLEMENTATIONS}

The results shows responses of the FrFT based Adaptive filter with LMs Algorithm and we applied a noised signal shown in Fig2 and compares the signal to noise ratio of Noised signal before and after the filtering for different Fractional Parameters of FrFT.

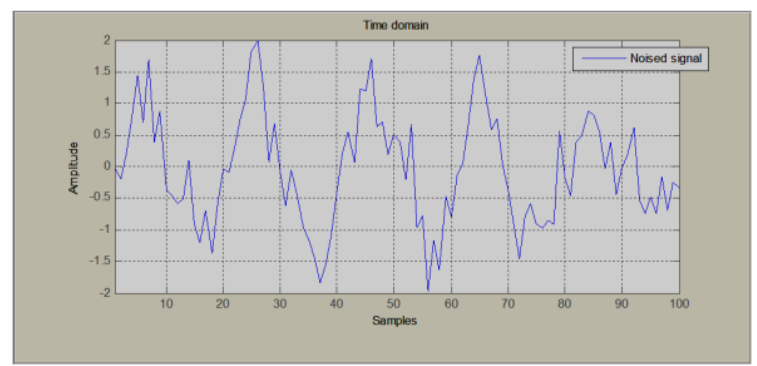

Fig2: Noised signal

When the Noised signal of fig2 is filtered with Adaptive Filter whole noise was removed, producing a near clean signal of fig:3 to fig: 8 with different window combinations of Desired FrFt based Filter. and SNR values of noised and denoised signals are calculated for LMs and FrFT based LMS Algorithms for different windows are shown in Table-1 to Table-5.

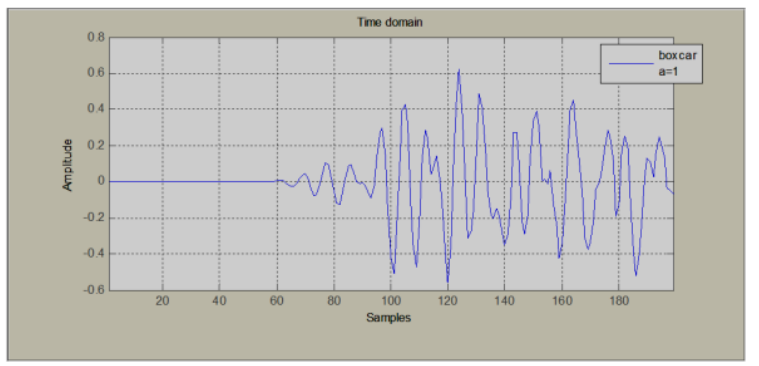

Fig3:Response of Adaptive filter with Rectangular window and $a=1$

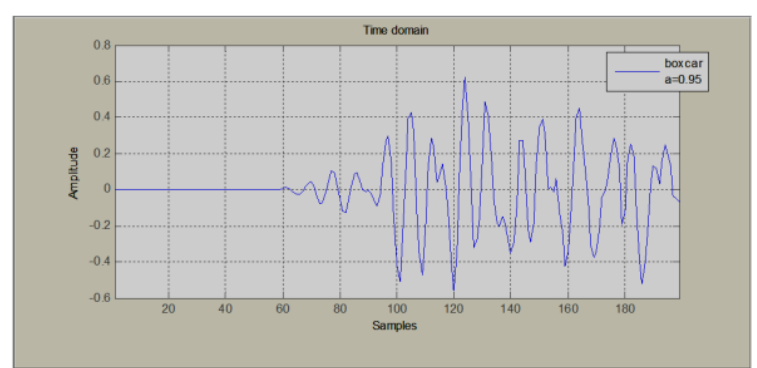

Fig4:Response of Adaptive filter with Rectangular window and $\mathbf{a}=\mathbf{0 . 9 5}$ 


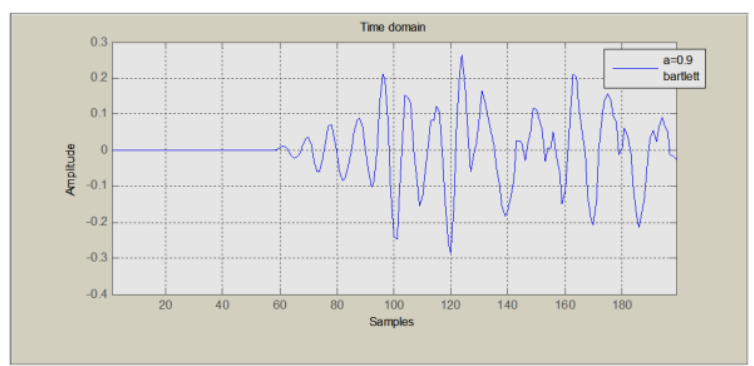

Fig5:Response of Adaptive filter with Bartlett window and $\mathbf{a}=0.9$

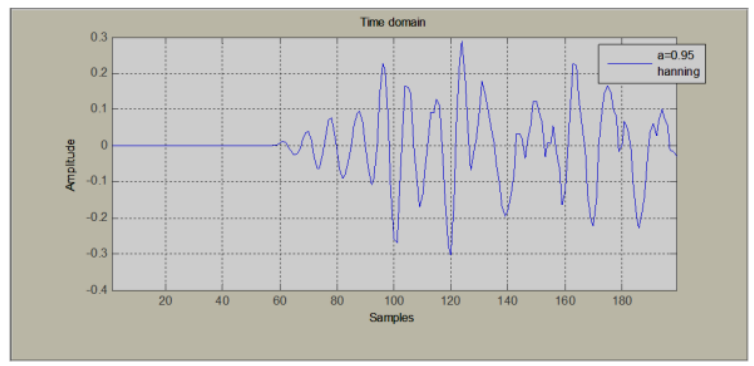

Fig6:Response of Adaptive filter with Hanning window and $\mathbf{a}=\mathbf{0 . 9 5}$

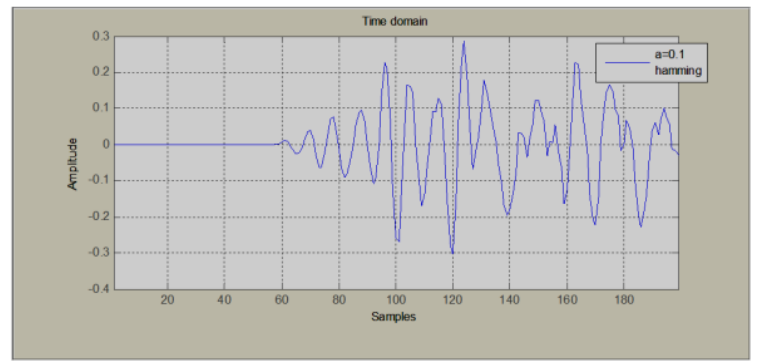

Fig7:Response of Adaptive filter with Hamming window and $\mathbf{a}=0.1$

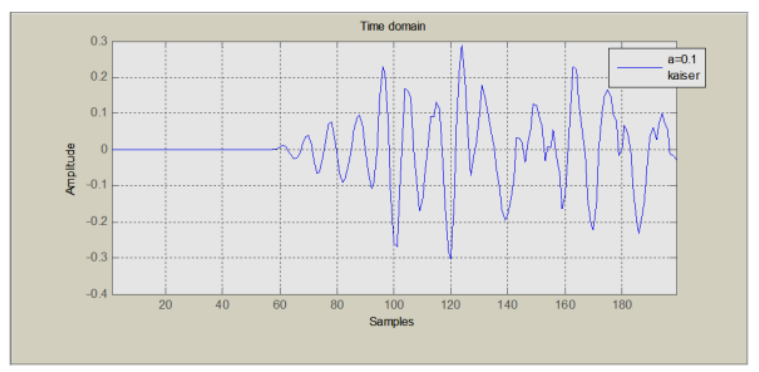

Fig8:Response of Adaptive filter with kaiser window and $\mathbf{a}=\mathbf{0 . 1}$

Table1: Response of FrFT+LMS based Rectangular window

\begin{tabular}{|l|l|l|l|}
\hline N & \multicolumn{2}{|c|}{ FrFT + LMS } & LMS \\
\cline { 2 - 4 } & $\begin{array}{c}\text { Tuning } \\
\text { parameter(a) }\end{array}$ & SNR in dB & SNR in dB \\
\hline 100 & $1^{*}$ & --- & 12.9578 \\
\hline
\end{tabular}

\begin{tabular}{|l|l|l|l|}
\hline 100 & 0.98 & 13.0029 & ---- \\
\hline 100 & 0.95 & 13.0184 & --- \\
\hline 100 & 0.9 & 12.9644 & --- \\
\hline 100 & 0.1 & 13.0194 & --- \\
\hline 100 & 0.01 & 12.9792 & ---- \\
\hline 100 & 0.05 & 13.0055 & ----- \\
\hline
\end{tabular}

Table2: Response of FrFT+LMS based Bartlett window

\begin{tabular}{|l|l|l|l|}
\hline \multirow{2}{*}{ N } & \multicolumn{2}{|c|}{ FrFT+LMS } & LMS \\
\cline { 2 - 4 } & $\begin{array}{c}\text { Tuning } \\
\text { parameter(a) }\end{array}$ & SNR in dB & SNR in dB \\
\hline 100 & $1^{*}$ & ---- & \\
\hline 100 & 0.98 & 11.4898 & ---- \\
\hline 100 & 0.95 & 11.4899 & --- \\
\hline 100 & 0.9 & 11.4905 & --- \\
\hline 100 & 0.1 & 11.4895 & --- \\
\hline 100 & 0.01 & 11.4898 & ---- \\
\hline 100 & 0.05 & 11.4896 & ---- \\
\hline
\end{tabular}

Table3: FT and FRFT Response of FrFT+LMS based hanning window

\begin{tabular}{|l|l|l|l|}
\hline \multirow{2}{*}{ N } & \multicolumn{2}{|c|}{ FrFT + LMS } & LMS \\
\cline { 2 - 4 } & $\begin{array}{l}\text { Tuning } \\
\text { parameter(a) }\end{array}$ & SNR in dB & SNR in dB \\
\hline 100 & $1^{*}$ & ---- & \\
\hline 100 & 0.98 & 12.9469 & ---- \\
\hline 100 & 0.95 & 12.9469 & --- \\
\hline 100 & 0.9 & 12.9469 & --- \\
\hline 100 & 0.1 & 12.9468 & --- \\
\hline 100 & 0.01 & 12.9468 & ---- \\
\hline 100 & 0.05 & 12.9462 & ---- \\
\hline
\end{tabular}


Table4:Response of FrFT+LMS based hamming window

\begin{tabular}{|l|l|l|l|}
\hline \multirow{4}{*}{ N } & \multicolumn{2}{|c|}{ FrFT + LMS } & LMS \\
\cline { 2 - 4 } & $\begin{array}{l}\text { Tuning } \\
\text { parameter(a) }\end{array}$ & SNR in dB & SNR in dB \\
\hline 100 & $1^{*}$ & & \\
\hline 100 & 0.98 & ---- & 12.9312 \\
\hline 100 & 0.95 & 12.9349 & ---- \\
\hline 100 & 0.9 & 12.9361 & --- \\
\hline 100 & 0.1 & 12.9318 & --- \\
\hline 100 & 0.01 & 12.9362 & --- \\
\hline 100 & 0.05 & 12.9329 & ---- \\
\hline
\end{tabular}

Table5: Response of FrFT+LMS based Kaiser window

\begin{tabular}{|l|l|l|l|}
\hline \multirow{2}{*}{ N } & \multicolumn{2}{|c|}{ FrFT + LMS } & LMS \\
\cline { 2 - 4 } & $\begin{array}{c}\text { Tuning } \\
\text { parameter(a) }\end{array}$ & SNR in dB & SNR in dB \\
\hline 100 & $1^{*}$ & ---- & \\
\hline 100 & 0.98 & 13.0292 & 13.0252 \\
\hline 100 & 0.95 & 13.0308 & ---- \\
\hline 100 & 0.9 & 13.0264 & --- \\
\hline 100 & 0.1 & 13.0305 & --- \\
\hline 100 & 0.01 & 13.0270 & ---- \\
\hline 100 & 0.05 & 13.0293 & ---- \\
\hline
\end{tabular}

\section{CONCLUSION}

The Implementation of Fractional Fourier Transform based Fir Filter using LMS Algorithm with Different Digital windows was performed. and we also applied a sample test noised signal to Adaptive filter and obtained denoised wave form at output which are shown in Fig-2 to Fig-8, and We compared SNR at input and Output for different window combinations which are shown from Table-1 to table-5.From the above discussions it is concluded that Fractional Fourier transform based Digital Filters with LMS Algorithm was given better Response in terms of SNR and Enhancement of Noise signal from noised input signal.

\section{REFERENCES}

[1] Saurabh Singh Rajput, Dr.S.S. BhadauriaImplementation Of Fir Filter Using Efficient Window Function And its Application In Filtering A Speech Signal-International Journal of Electrical,Electronics and mechanical control.
[2] Sonika Gupta, Aman Panghal- Performance Analysis of FIR Filter Design by Using Rectangular, Hanning and Hamming Windows Methods- International Journal of Advanced Research in Computer Science and Software Engineering- Volume 2, Issue 6, June 2012- ISSN: 2277 $128 \mathrm{X}$

[3] TAO ZHANG- RESEARCH ON DESIGN FIR DIGITAL FILTER USING MATLAB AND WINDOW FUNCTION METHOD- Journal of Theoretical and Applied Information Technology- 10th February 2013. Vol. 48 No.1

[4] R.W.Schafer,L.R.Rabiner,O.Herrmann-"FIR digital filter banks for speech analysis"-The Bell system Technical Journal-vol:54,no:3, march 1979.

[5] Mridula Malhotra, The Performance Evaluation of Window Functions and Application to FIR Filter DesignInternational Journal of Scientific \& Engineering Research, Volume 2, Issue 12, December-2011 1 ISSN 2229-5518

[6] J. Parak, J. Havlik-ECG Signal Processing And Heart Rate Frequency Detection Methods

[7] P Bonizzi, O Meste, V Zarzoso-Spectral analysis of atrial signals directly from surface ECG exploiting compressed spectrum.

[8] LEIF SO RNMO, PABLO LAGUNAELECTROCARDIOGRAM (ECG) SIGNAL PROCESSING.

[9] R. Sassi, L.T. Mainardi, P. Maison-Blanche3 and S. Cerutti-ESTIMATION OF SPECTRAL PARAMETERS OF RESIDUAL ECG SIGNAL DURING ATRIAL FIBRILLATION USING AUTOREGRESSIVE MODELS.

[10] CN Nowak, G Fischer, L Wieser, B Tilg, HU Strohmenger-Frequency Spectrum of the Intracardiac and Body Surface ECG during Ventricular Fibrillation - a Computer Model Study

[11] Jong-Yih Lin _, Ching-Wen Liao "New IIR filter-based adaptive algorithm in active noise control applications: Commutation error-introduced LMS algorithm and associated convergence assessment by a deterministic approachI"- Automatica 44 (2008) 2916_2922-Elsevier Publications

[12] Ibtissam Constantin,Regis Lengelle-"performance Analysis of Kernal Adaptive Filters Based on LMS Algorithm"-procedia Computer science 20(2013) 3945 ,sciencdirect.

[13] Michael A. Vaudrey, William T. Baumann, William R. Saunders-"Stability and operatingconstraints of adaptive LMS-based feedback control"-Automatics-Sciencdirect

[14] Wang An-dong, Liu Lan ,Wei Qin-“An Adaptive Morphologic Filter Applied to ECG De-noising and Extraction of R Peak at Real-time" 2012 AASRI Conference on Computational Intelligence and Bioinformatics,Elsevier

[15] Kaibo $\mathrm{Hu}^{*}$, Yaxuan Liu-“Adaptive Noise Cancellation Method for Fiber Optic Gyroscope"- 2012 International Workshop on Information and Electronics Engineering (IWIEE),Elsevier

[16] Namias.V-“The FrFT and Time Frequency 
representation"-J.Inst.Math.Applications,Vol:25,pp.241265,1980 .

[17] Soo-Chang pei,Mon Hung Yeh,TzyyliangLuo"Fractional Fourier Series Expansion for Finite signals and Dual Extension to Discrete-Time Fractional Fourier Transform"-IEEE Transactions on signal processing,vol:47,No:10,1999.

[18] Soo-Chang pei - "Two-Dimensional Affine generalized Fractional Fourier Transform"-IEEE Transactions on signal processing,vol: 49,No:4,April 2009

[19] I.SamilYetik,M.AlperKutay, Haldun .M.Ozaktas-"Image representation and compression with the Fractional Fourier Transform", Optics communications 197(2001),275-278,Elsevier publications.
[20] V.AshokNarayanan,K.M.M.Prabhu-“The

FrFT:Theory,Implementation and error Analysis", Microprocessor and Microsystems 27(2003),511521,Elsevier Publications.

[21] Ran Tao, Kiang-Yi meng,Yue Wang-“Image encryption with multi orders Fractional Fourier Transforms",IEEE transactions on Information Forensics and Security,vol:05, No:04,December 2010.

[22] P.V.Muralidhar,A.S.SrnivasaRao,Dr.S.K.Nayak-Spectral Interpretation of Sinusoidal wave using Fractional Fourier Transform based FIR window functions",vol:04,N0:6,PP:652-657,International Review on computers and software, November 2009. 